implementation of HIPEC with the CO2 PRS, probably due to a better drug distribution in the peritoneal cavity. This is of critical importance given that pattern of recurrence as carcinomatosis is undoubdtely associated with unfavourable outcome.

\section{EPV227/\#79 OVARIAN CANCER INCIDENCE AFTER BILATERAL SALPINGO-OOPHORECTOMY IN WOMEN WITH HISTOLOGICAL PROVEN ENDOMETRIOSIS OR ADENOMYOSIS}

${ }^{1} \mathrm{M}$ Hermens ${ }^{*},{ }^{2} \mathrm{~A}$ Van Altena, ${ }^{3} \mathrm{~J}$ Bulten, ${ }^{1} \mathrm{H}$ Van Vliet, ${ }^{4} \mathrm{~A}$ Siebers, ${ }^{1} \mathrm{R}$ Bekkers. ${ }^{1}$ Catharina Hospital, Obstetrics and Gynaecologt, Eindhoven, Netherlands; ${ }^{2}$ Radboudumc, Obstetrics and Gynaecology, Nijmegen, Netherlands; ${ }^{3}$ Radboudumc, Pathology, Nijmegen, Netherlands; ${ }^{4}$ PALGA, Pathology, Houten, Netherlands

\subsection{6/ijgc-2021-IGCS.298}

Objectives Endometriosis is associated with an increased ovarian cancer incidence. Surgical treatment of endometriosis might reduce this risk. Therefore, we assessed the ovarian cancer incidence in women with endometriosis after bilateral salpingo-oophorectomy (BSO).

Methods All women with histological proven endometriosis between 1990 and 2015 in the Netherlands were identified. Women with a BSO without ovarian cancer at time of surgery were selected as cases $(n=14,410)$. We selected two control cohorts; 1) women with histological proven endometriosis without BSO or with ovarian cancer at time of BSO $(n=115,323)$, and 2) women with a benign dermal nevus $(n=132,654)$. Histological diagnoses of ovarian or extra-ovarian cancers were retrieved. Incidence rate ratios (IRR) were estimated for (extra) ovarian cancer.

Results We identified $13(0.09 \%)$ extra-ovarian cancers in the BSO cohort and 2,036 (1.8\%) and $471(0.4 \%)$ ovarian cancers in the endometriosis and nevus cohort, respectively. We found an age-adjusted IRR of 0.02 (95\%CI 0.01-0.04) when the BSO cohort was compared with the endometriosis cohort and an age-adjusted IRR of 0.20 (95\%CI 0.11-0.37) when comparing the BSO to the nevus cohort (table 1 ). Median age at cancer diagnosis was 61 (IQR 56-74) in the BSO cohort, 55 (IQR 48-63) in the endometriosis cohort and 58 years (IQR 51-65) in the nevus cohort (both $\mathrm{p}<0.05$ ).

Conclusions We found a significantly reduced (extra-)ovarian cancer incidence in women with endometriosis and a

Abstract EPV227/\#79 Table 1 Estimated incident rates per 100,000 person-years, crude incidence rate rations, and ageadjusted incidence rate ratios of ovarian cancer in women with endometeriosis with BSO compared to 1) women with endometriosis without BSO (or BSo at time of ovarian cancer) and 2) women with a being dermal nervus

\begin{tabular}{|c|c|c|c|}
\hline & $\begin{array}{c}\text { Incid ence rate per } 100,000 \\
\text { person-years }(95 \% \text { CJ) }\end{array}$ & $\begin{array}{c}\text { Crude incidence rate } \\
\text { ratios }(95 \% 6 \mathrm{CI}) \\
\end{array}$ & $\begin{array}{l}\text { Age-adjusted incidence } \\
\text { rate ratios }(95 \% \mathrm{CI})\end{array}$ \\
\hline BSO & $6.47(3.75-11.14)$ & - & - \\
\hline $\begin{array}{l}\text { Endometriosis } \\
\text { without BSO }\end{array}$ & $118.40(113.33-123.57)$ & $0.05(0.03-0.09)$ & $0.02(0.01-0.04)$ \\
\hline Nevus & $23.21(21.20-25.40)$ & $0.28(0.15-0.48)$ & $0.20(0.11-0.37)$ \\
\hline
\end{tabular}

BSO when compared to both controls with endometriosis without BSO, and controls without histological proven endometriosis.

\section{EPV228/\#80 INCREASED INCIDENCE OF OVARIAN CANCER IN BOTH ENDOMETRIOSIS AND ADENOMYOSIS}

${ }^{1} \mathrm{M}$ Hermens ${ }^{*},{ }^{2} \mathrm{~A}$ Van Altena, ${ }^{3} \mathrm{~J}$ Bulten, ${ }^{1} \mathrm{H}$ Van Vliet, ${ }^{4} \mathrm{~A}$ Siebers, ${ }^{1} \mathrm{R}$ Bekkers. ${ }^{1}$ Catharina Hospital, Obstetrics and Gynaecologt, Eindhoven, Netherlands; ${ }^{2}$ Radboudumc, Obstetrics and Gynaecology, Nijmegen, Netherlands; ${ }^{3}$ Radboudumc, Pathology, Nijmegen, Netherlands; ${ }^{4}$ PALGA, Pathology, Houten, Netherlands

\subsection{6/ijgc-2021-IGCS.299}

Objectives Recently we conducted a study in which we found an increased association of ovarian cancer in women with endometriosis. Analyses showed that the cohort included both women with endometriosis externa and adenomyosis. Therefore, in the present study we assessed the association between endometriosis and/or adenomyosis and ovarian cancer.

Methods We identified all women with histological proven endometriosis (51,544 women) and/or adenomyosis (85,015 women) from the Dutch pathology database (1990-2015) and matched them with women with a benign dermal nevus (132,654 women). Histology results for ovarian cancer were retrieved. We estimated crude and age-adjusted incidence rate ratios (IRR) for ovarian cancer.

Results We found 1,017 (2.0\%), 1,284 (1.5\%) and 471 $(0.4 \%)$ ovarian cancer cases in the endometriosis, adenomyosis and nevus cohort, respectively. The age-adjusted IRRs were 19.75 (95\%CI 16.70-23.35) in the endometriosis cohort and 5.93 (95\%CI 4.91-7.16) in the adenomyosis cohort (table 1). The highest IRRs were found for endometrioid and clear cell ovarian cancer subtypes (table 1). Excluding the first year of follow-up did not result in a significant IRR for ovarian cancer overall but resulted in a statistically significant IRRs for clear cell and endometrioid ovarian cancer (table 1).

Conclusions We found an increased ovarian cancer incidence in both histological proven endometriosis and adenomyosis. This increased incidence was largest for endometriosis. Excluding the first year of follow-up resulted in an increased incidence for endometrioid ovarian cancer in both cohorts and clear cell ovarian cancer in the endometriosis cohort.

Abstract EPV228/\#80 Table 1 Observed number of ovarian cancers, estimated incidence rate per 100,000 person-years, crude incidence rate ratios and age-adjusted incidence. Rate ratios of ovarian cancers of women with endometriosis or adenomyosis compared with a benign dermal nevus, per ovarian cancer subtype and overall

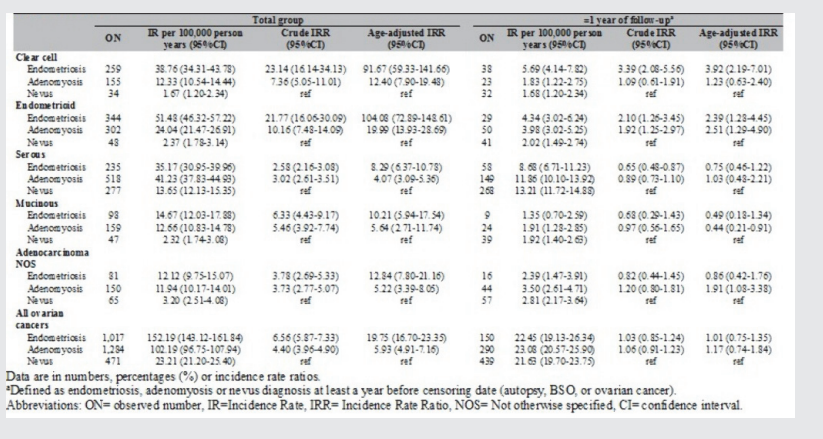

